\title{
Non-Invasive Visualization of Ferroelectric Domain Structures on the Non-Polar y-Surface of $\mathrm{KTiOPO}_{4}$ via Raman Imaging
}

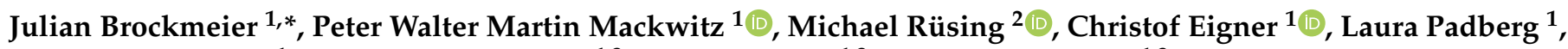 \\ Matteo Santandrea ${ }^{1}$, Christine Silberhorn ${ }^{1,3}$, Artur Zrenner ${ }^{1,3}$ and Gerhard Berth ${ }^{1,3}$ \\ 1 Department of Physics, University of Paderborn, Warburger Strasse 100, 33098 Paderborn, Germany; \\ peterm@mail.upb.de (P.W.M.M.); christof.eigner@upb.de (C.E.); laura.padberg@upb.de (L.P.); \\ matteo.santandrea@upb.de (M.S.); christine.silberhorn@upb.de (C.S.); artur.zrenner@upb.de (A.Z.); \\ berth@physik.upb.de (G.B.) \\ 2 Institute of Applied Physics, Technical University Dresden, Nöthnitzer Straße 61, 01187 Dresden, Germany; \\ michael.ruesing@tu-dresden.de \\ 3 Center for Optoelectronics and Photonics (CeOPP), Warburger Strasse 100, 33098 Paderborn, Germany \\ * Correspondence: julian.brockmeier@uni-paderborn.de
}

Citation: Brockmeier, J.; Mackwitz, P.W.M.; Rüsing, M.; Eigner, C.; Padberg, L.; Santandrea, M.; Silberhorn, C.; Zrenner, A.; Berth, G. Non-Invasive Visualization of Ferroelectric Domain Structures on the Non-Polar y-Surface of $\mathrm{KTiOPO}_{4}$ via Raman Imaging. Crystals 2021, 11, 1086. https://doi.org/10.3390/ cryst11091086

Academic Editors: Guisheng $\mathrm{Xu}$ and Yizheng Tang

Received: 6 August 2021

Accepted: 3 September 2021

Published: 7 September 2021

Publisher's Note: MDPI stays neutra with regard to jurisdictional claims in published maps and institutional affiliations.

Copyright: (c) 2021 by the authors. Licensee MDPI, Basel, Switzerland. This article is an open access article distributed under the terms and conditions of the Creative Commons Attribution (CC BY) license (https:// creativecommons.org/licenses/by/ $4.0 /)$.

\begin{abstract}
Potassium titanyl phosphate (KTP) is a nonlinear optical material with applications in high-power frequency conversion or quasi-phase matching in submicron period domain grids. A prerequisite for these applications is a precise control and understanding of the poling mechanisms to enable the fabrication of high-grade domain grids. In contrast to the widely used material lithium niobate, the domain growth in KTP is less studied, because many standard methods, such as selective etching or polarization microscopy, provides less insight or are not applicable on non-polar surfaces, respectively. In this work, we present results of confocal Raman-spectroscopy of the ferroelectric domain structure in KTP. This analytical method allows for the visualization of domain grids of the non-polar KTP y-face and therefore more insight into the domain-growth and -structure in KTP, which can be used for improved domain fabrication.
\end{abstract}

Keywords: Raman spectroscopy; KTP; periodic poling; ferroelectric domains; Raman imaging

\section{Introduction}

Potassium titanyl phosphate $\left(\mathrm{KTiOPO}_{4}, \mathrm{KTP}\right)$ is a nonlinear optical material that belongs to the point group $m m 2$ and the space group Pna2 $2_{1}$ [1], offering a wide transparency window, as well as large electro-optic and nonlinear constants [2,3]. Additional advantages are the low photorefractivity [3] and the high damage threshold.

More recently, it was demonstrated that KTP allows for the fabrication of ultra-short ferroelectric domain grids [4-6], i.e., submicron periods. These find application in efficient frequency conversion interfaces between the UV and mid IR that link photonics and solid-state quantum systems [6], mirrorless optical parametric oscillators [7], or counterpropagating parametric down-conversion (PDC) [8]. The latter provides spectrally decorrelated photons with narrower bandwidths and at higher source brightness than could be achieved with standard forward-propagating PDC and spectral filtering [8]. Efficient frequency conversion processes require phase matching between the interacting waves, which is typically achieved by employing the quasi-phase matching (QPM) technique by fabricating periodically poled ferroelectric domain structures $[9,10]$. The main advantage of QPM over other phase matching schemes, such as birefringence phase matching or modal phase matching, is that QPM allows for phase matching of the conversion process independent from the material properties (birefringence) or waveguide design, hence allowing for optimizing the different properties for best conversion efficiencies [11]. Furthermore such a design allows for adressing the highest nonlinear coefficient $d_{33}$ [12]. The challenge, however, is to fabricate a homogeneous domain structure. The usual method for this is 
electric field poling. Electric field poling of KTP is more challenging than the poling of lithium niobate (LN) $[13,14]$ mainly due to the large ionic conductivity observed along the polar axis of KTP [15]. On the one hand, this conductivity makes it demanding to maintain high electric fields on large poling areas, while on the other hand the ionic current at the same time masks the typical ferroelectric switching current [13], which is used in other materials, such as $\mathrm{LN}$, as a convenient measure for the poling progress. Even more, in some cases this ionic conductivity has often been observed to spatially vary over flux grown wafers [13], which is connected to a spatially dependent potassium deficiency often inevitably introduced during growth. This led to the development of novel poling and monitoring schemes for KTP different from traditional LN approaches $[10,16]$. To optimize and understand domain fabrication in KTP and any other material, the assessment and analysis of the transferred domain structure is imperative. However, studies of the ferroelectric domain structure, the underlying poling mechanism and domain growth in depth, i.e., domain cross-sections on non-polar $\mathrm{x}$ - and $\mathrm{y}$-surfaces, are sparsely studied in KTP $[13,17]$. The standard method to visualize the domain structures is selective chemical etching; however, our own experiments, as well as previous works by Canalias et al. [17], show that this method cannot be employed to visualize domain structures on the non-polar surfaces. Another standard method to analyze domain structures is Piezo-response force microscopy (PFM) [18]. This method can be conveniently employed to study domain structure on polar and non-polar surfaces on KTP. As an AFM derivative, it offers nanometer scale spatial resolution, which is mostly limited by the tip size. However, it is mostly a surface sensitive method. Most domains can be detected up to $1.7 \mu \mathrm{m}$ depth [19]. Noninvasive optical microscopy methods, such as Raman imaging $[20,21]$ or second harmonic microscopy [22-25], offer an additional depth discrimination [26-28] and are sensitive to other material properties, such as stoichiometry [29], defects [30] or crystal symmetry changes [31,32]. Therefore, these methods offer a route to study interaction between domain structure and stoichiometry or strain, which potentially plays an important role in $\mathrm{Rb}$-exchanged waveguides in KTP [33]. Recently, we investigated the Raman imaging contrast mechanism of ferroelectric domain walls (DW) [34]. In the present study, we apply this knowledge to investigate and image DWs on the non-polar surfaces of KTP to gain more insight into the domain growth mechanism as well as investigate the reliability of the utilized poling monitoring based on the electro-optic effect.

\section{Experimental Design}

A commercially obtained flux grown single-crystalline and mono-domain KTP wafer is diced into pieces of dimensions of typically $10 \mathrm{~mm}$ by $6 \mathrm{~mm}$ by $1 \mathrm{~mm}$ (X by Y by Z). The potassium stoichiometry of flux grown KTP has previously been observed to vary over the wafer scale, which influences the local poling behavior. The poling structure is transferred to the sample by a standard optical lithography process (photo resist patterned with $7.6 \mu \mathrm{m}$ period on the $-\mathrm{z}$ face) as schematically shown in Figure 1. Here, the long axis of the periodically domains are oriented parallel to the $y$-direction. The $-\mathrm{z}$ and $+\mathrm{z}$ surfaces are then electrically contacted by an electrolyte and a metal back electrode, respectively. The domain inversion is achieved by applying several high voltage pulses generated via a pulse generator with an attached high-voltage amplifier (Trek 20/20C). The same poling process is used to fabricate samples suitable for a $\mathrm{Rb}$-exchange process to obtain periodically poled Rb:KTP waveguides [35]. Typical waveguides have depths of less than $10 \mu \mathrm{m}$. It is imperative that the design parameters of the domain structure are met close to the surface intended for the waveguide fabrication. To monitor the poling progress we employ an all optical monitoring method based on the electro-optic effect developed by Karlsson et al. [10,16]. We place a weakly focused laser beam within 100 to $200 \mu \mathrm{m}$ close to the $-\mathrm{z}$ surface. The laser beam is linearly polarized at $45^{\circ}$ with respect to the $\mathrm{z}$-axis. As KTP is birefringent, the laser will exit the crystal at an arbitrarily, elliptically polarized state. Via a quarter wave-plate the light polarization is linearized, directed to another linear polarizer and detected by a photo-diode. If the voltage is increased the light field 
component polarized parallel to the z-axis will see a voltage-dependent phase shift due to the linear electro-optical effect (Pockel's effect) and the detected signal intensity will oscillate with linearly increasing voltage as discussed in detailed by Karlsson et al. [14]. Depending on the domain orientation, the refractive index will be increased or decreased due ot the electro-optic effect. This is because the sign of the electro-optical tensor is reversed in domains of opposing orientation. This means when half of the domains are inverted along the light path, i.e., in an ideal case a 50/50 duty cycle is reached, as shown in Figure 1c, the voltage dependent oscillation will vanish, because there is no net phase shift for the light component parallel to the z-axis. We apply voltage pulses to the sample until this point is reached.

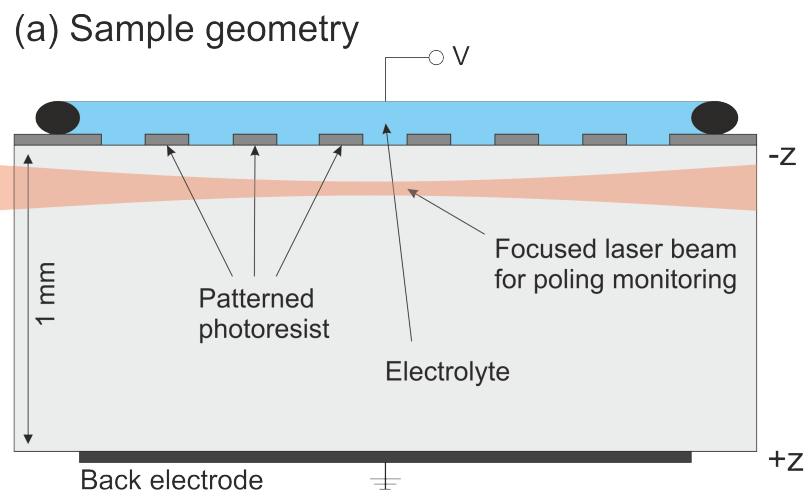

(c) Poling stop

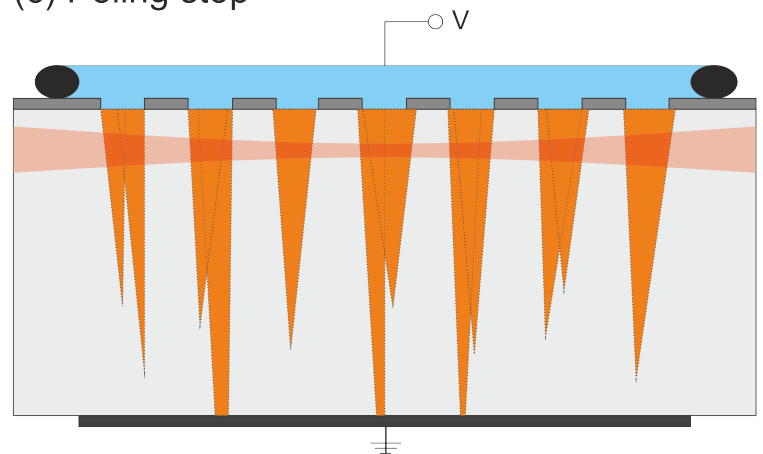

(b) Poling start

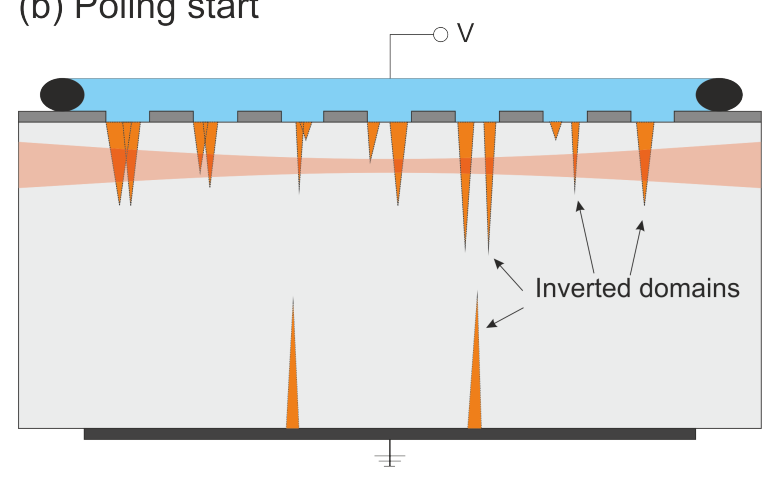

(d) Raman imaging geometries

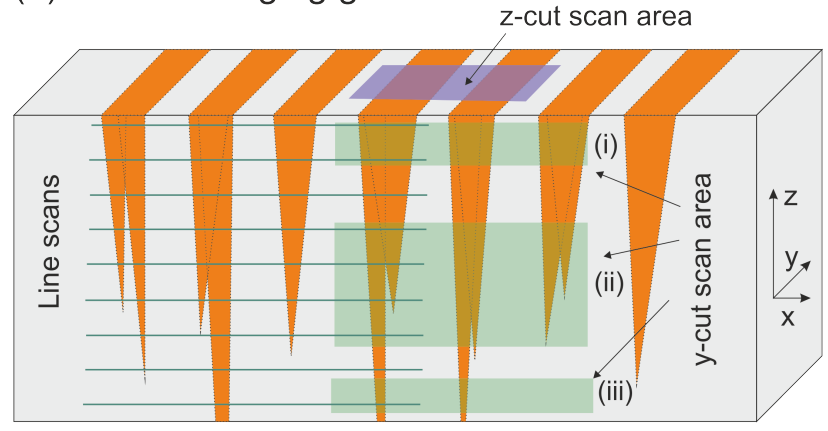

Figure 1. Principle sketch of: (a) Poling and monitoring geometry used in this study. (b) Poling start: With application of the first pulse over the coercive field, inverted domains will nucleate under the electrically contacted area. (c) Poling stop: After application of multiple pulses, the domains will have grown deeper and have merged until an approximately 50/50 duty cycle in the monitored regions is achieved. (d) Geometries for the presented Raman analysis. Figure is not to scale.

Domain reversal is microscopically subdivided into four steps [36,37]: (1) nucleation of new domains on one or two of the polar surfaces, (2) forward growth of the nucleated, switched domains along the z-axis, (3) sideways spreading in the $\mathrm{x}$ - and $\mathrm{y}$-plane, and (4) coalescence of neighboring domains. Domain propagation speeds are characterized by a substantial directional dependence. In KTP for forward growth, typical values between 50 and $200 \mu \mathrm{m} / \mathrm{s}$ are observed [13]. However, in the y-direction, the domain propagation speed is between 10 and $30 \mu \mathrm{m} / \mathrm{s}$, while it is only 0.2 to $1.2 \mu \mathrm{m} / \mathrm{s}$ in the x-direction [13]. The two to three orders of magnitude difference between forward and sideways growth is among one of the reasons why KTP is a suitable material for fabrication of ultra-short domain periods $[5,7]$.

When the monitoring indicates that $50 \%$ of the electrically contacted area is flipped, poling is stopped. This is sufficient, for example, to achieve homogeneously poled surfacenear waveguides [38]. However, we expect only incomplete domain inversion in the deeper regions of the crystals, as schematically indicated in Figure 1c. Raman measurements were performed incident from $\mathrm{z}$-face via light oriented parallel to the crystallographic c-axis and 
incident y-face via light perpendicular to the c-axis. As previously established [34], both allow for a domain wall contrast. In each case, the surface-near interaction provides the best resolution possible. Alternatively, the confocal mode allows for imaging the cross-section (y-face) by gradually focusing deeper into the material from the $\mathrm{z}$-face. However, in this case, a refracting (and in this case even a birefringent) medium will lead to a gradual decrease in intensity as well as a gradual decrease in resolution due to the increasing distortion of the focus field. Therefore, such scans may be limited to depths closer to the respective surface, e.g., $<100 \mu \mathrm{m}$, which is suitable for the analysis of (periodically poled) waveguides. The Raman analysis was performed on a custom built confocal setup, which utilizes excitation light of $532 \mathrm{~nm}$ at $50 \mathrm{~mW}$ focused via an infinity-corrected objective $(\mathrm{NA}=0.7)$ on the sample. The scattered light is collected by the same objective and directed to the spectrometer (KOSI Holospec, f/1.8i) with attached CCD camera (Andor Newton, BI) for detection. Spectral suppression of the Rayleigh scattered light is provided by a dichroic mirror separating excitation and detection path and an appropriate Notch filter integrated in the spectrometer unit. A pinhole of $10 \mu \mathrm{m}$ diameter is inserted in the detection path providing in combination with the objective lens a spatial resolution of $<400 \mathrm{~nm}$ in lateral direction and $<2000 \mathrm{~nm}$ in axial direction. With respect to a fixed laser focus, the confocal application demands raster scanning via $3 \mathrm{D}$ positioning of the sample to obtain an image. In our setup, the scanning is performed with respect to a fixed focus via a nanopositioning stage with $2 \mathrm{~nm}$ resolution and $180 \mu \mathrm{m}$ by $180 \mu \mathrm{m}$ by $20 \mu \mathrm{m}$ scan range (Tritor, Piezosystem Jena). This piezo-stage is mounted on a custom-built long range positioning system with $40 \mathrm{~mm}$ by $40 \mathrm{~mm}$ in lateral direction and $25 \mathrm{~mm}$ in axial direction. This long range stage provides a $\mu \mathrm{m}$-scale accuracy over the whole range. In particular, the latter stage is important for this study, as it allows us to study the domain structure over a large area in three dimensions and repeatably select areas of interest for high resolution imaging. Further details on the system can be found in previous work [39].

\section{Raman Analysis on KTP}

As known from previous works, the Raman spectrum is modified by the presence of DWs [34]. This comprises intensity variations, changes in peak position or fullwidth-at-half-maximum (FWHM) and depends on the specific phonon, as indicated in Figure 2. A spatial mapping of these spectral features allows for the visualization of domain structures.

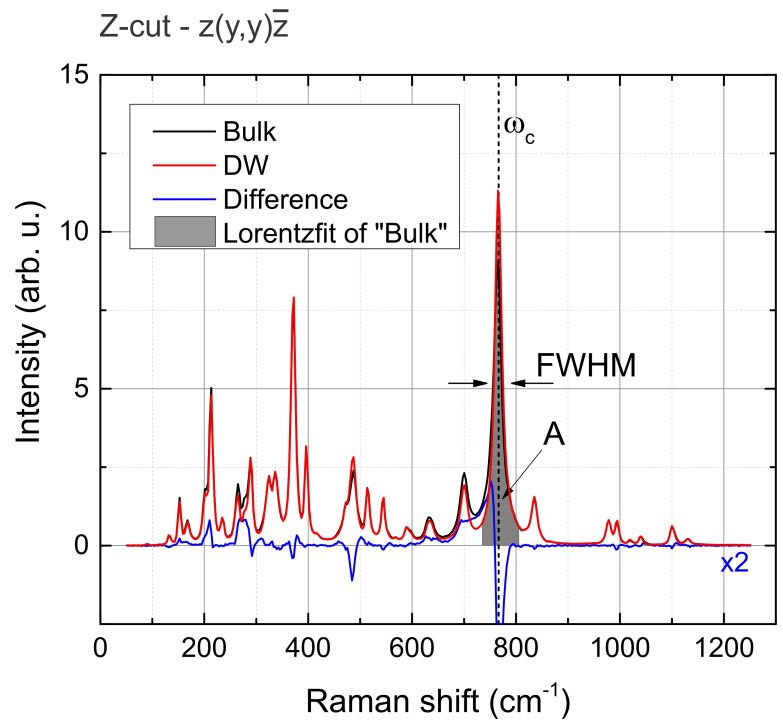

Figure 2. Raman spectra of a DW compared to bulk spectra for the scattering geometry $z(y, y) \bar{z}$. For better visibility of the changes, a difference spectrum is given. 
In Figure 2, the as-measured Raman spectrum of bulk KTP compared to a spectrum taken at a DW measured for z-incident is shown. The scattering geometry can be conveniently described in Porto's notation, here for example $\mathrm{z}(\mathrm{y}, \mathrm{y}) \overline{\mathrm{z}}$, where the symbols in the brackets denote the polarization of the incoming and outgoing light in crystal coordinates, while the outer symbols denote the propagation direction of the incident light $(\mathrm{z})$ and detected light $(\bar{z})$. The presented bulk and DW spectra only show minor differences, with intensity changes for certain modes in the order of a few percent and some peak-shifts by a few $\mathrm{cm}^{-1}$. For both scattering geometries the differences between the bulk and DW spectra are small, which can be partly explained by the diffraction-limited optical resolution $>400 \mathrm{~nm}$ and the size of a DW, which is supposed to be only a few nm down to a unit cell large [40]. To better highlight the changes, the corresponding difference spectrum is shown. In the literature, the changes in the Raman spectra of DWs are explained by two mechanisms. On the one hand, ferroelectric DWs are expected to be accompanied by strain $[40,41]$. Therefore, inner electric fields are generated via piezo-electricity, which influences the Raman data [42-44]. This is referred to as the elasto- and electro-optic coupling [45]. The second mechanism assumes changes in the Raman selection rules according to Stone and Dierolf [46]. Here, the DW is understood as a planar defect. A planar defect is accompanied by a defect momentum due to the step-wise change in crystal structure. Therefore, in the presence of DWs phonons propagating at angles non-parallel to the incident beam may be present in the measured spectrum. If the spectrum of these phonons propagating at oblique angles differs from the bulk spectrum, a change at the DW spectrum is observed [46,47]. Based on the model by Stone and Dierolf [46], we have recently shown that the DW spectrum of KTP can be explained and predicted by measuring the angular dependency of the spectrum [47]. For example, the dominating peak in Figure 2 is an $\mathrm{A}_{1}$-LO type, which shifts to lower wavenumbers at higher phonon propagation angles resulting in a spectral intensity increase in the $69-700 \mathrm{~cm}^{-1}$ range. Due to the diffraction-limited spatial resolution, the spectrum measured at a DW is always a sum of the DW spectrum and a bulk spectrum. In total this explains the decrease in intensity of this mode, the shift to lower wave-numbers, as well the observed slight broadening as bulk and DW spectra are detected simultaneously.

Based on our spectral analysis we performed Raman imaging in terms of the spatial variation of the three parameters: intensity, FWHM and peak frequencies for specific phonons. As an example, the corresponding results for the dominating phonon around $760 \mathrm{~cm}^{-1}$ are shown in Figure 3. Here, for z-cut an intensity decrease (Figure 3a), broadening of linewidth (Figure $3 b$ ) and a decrease in wave number by more than $1 \mathrm{~cm}^{-1}$ is observed (Figure 3c). For all three fitting parameters, the domain structure becomes clearly visible with the targeted domain period $(7.6 \mu \mathrm{m})$ and duty cycle. A comparison of the three images shows that plotting the peak shift provides the best results for visualization of DWs, because peaks shifts are not susceptible for slight intensity variations, e.g., by surface irregularities.

The focus of this work lies on the visualization of the ferroelectric domain structure on the non-polar y-face of KTP. Therefore, we performed a spectral analysis to reveal fingerprints of phonon modes sensitive to the domain wall. It should be noted, that in $\mathrm{y}$-face KTP only for the crossed polarization configuration a strong DW contrast is expected, as only these spectra show a directional dependency [34]. Therefore, the Raman spectrum of the DW is compared to the bulk spectrum as shown in Figure 4 for the $y(x, z) \bar{y}$ scattering geometry. 
Z-cut KTP: $760 \mathrm{~cm}^{-1}$ phonon-mode

(a) Intensity
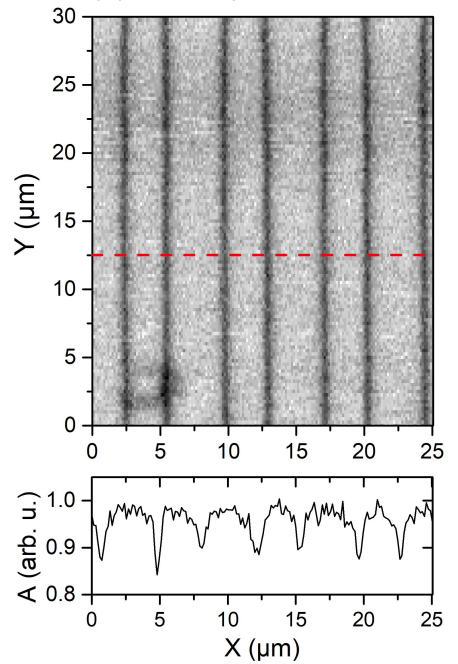

(b) FWHM
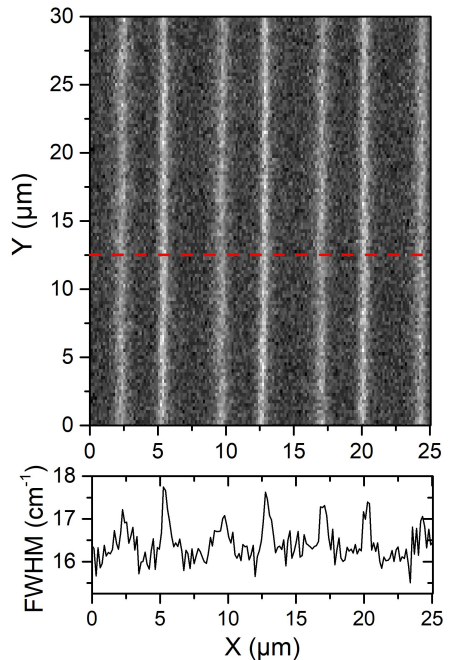

(c) Peak wavenumber
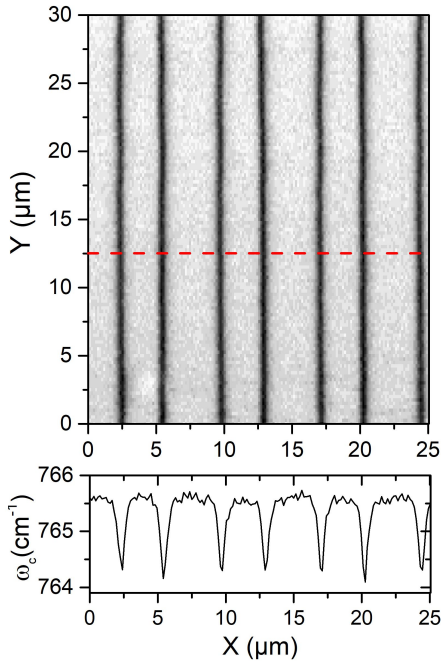

Figure 3. Raman images of the -z-surface of a periodically poled KTP sample based on the variation of intensity (a), FWHM (b) and peak wave numbers (c) of the $760^{-1}$ phonon mode highlighted in Figure 2.

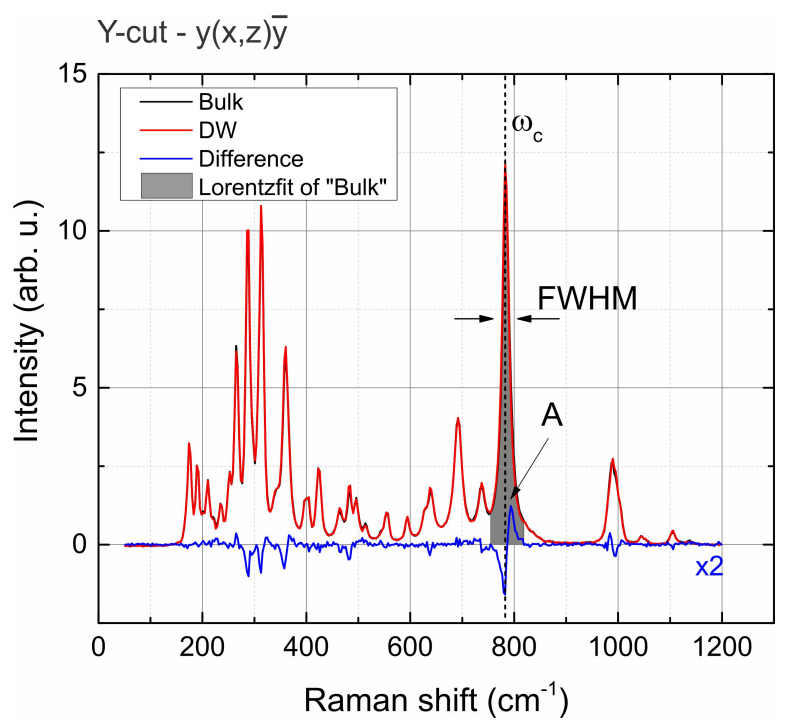

Figure 4. Raman spectra of a DW compared to bulk spectra for the scattering geometry $y(x, z) \bar{y}$. For better visibility of the changes a difference spectrum is given.

Corresponding structure sensitive phonon modes are listed with respect to changes in intensity, FWHM and peak frequency in Table 1 in order to provide a phonon mode assignment. The $287 \mathrm{~cm}^{-1}$ mode is assigned to the $v_{5}\left(\mathrm{TiO}_{6}\right)$ vibration. The neighboring $313 \mathrm{~cm}^{-1}$ mode also belongs to the same crystallographic structure, but is related to the $v_{4}\left(\mathrm{TiO}_{6}\right)$ vibration according to literature, whereas the $991 \mathrm{~cm}^{-1}$ mode is linked to the $v_{1}$ vibration of the $\mathrm{PO}_{4}$ tetrahedron. 
Table 1. Comparison of the intensity, FWHM and Raman shift of the peak frequency of the measured spectra in $y(x, z) \bar{y}$ scattering geometry of bulk KTP and a KTP DW. Positive change indicates, respectively, a gained intensity, FWHM or shift to higher frequencies, whereas a negative value indicates a lowered intensity, FWHM or a shift to lower frequencies.

\begin{tabular}{|c|c|c|c|c|c|c|c|c|c|}
\hline $\begin{array}{l}\text { Mode } \\
\mathbf{y}(\mathrm{x}, \mathrm{z}) \overline{\mathrm{y}} \\
\left(\mathrm{cm}^{-1}\right)\end{array}$ & $\begin{array}{c}\text { Intensity } \\
\text { Bulk } \\
\text { (a.u.) }\end{array}$ & $\begin{array}{c}\text { Intensity } \\
\text { DW } \\
\text { (a.u.) }\end{array}$ & $\begin{array}{l}\Delta_{\text {Int. }} \\
(\%)\end{array}$ & $\begin{array}{c}\text { FWHM } \\
\text { Bulk } \\
\left(\mathrm{cm}^{-1}\right)\end{array}$ & $\begin{array}{c}\text { FWHM } \\
\text { DW } \\
\left(\mathrm{cm}^{-1}\right)\end{array}$ & $\begin{array}{c}\Delta_{\mathrm{FWHM}} \\
\left(\mathrm{cm}^{-1}\right)\end{array}$ & $\begin{array}{c}\text { Shift } \\
\text { Bulk } \\
\left(\mathrm{cm}^{-1}\right)\end{array}$ & $\begin{array}{c}\text { Shift } \\
\text { DW } \\
\left(\mathrm{cm}^{-1}\right)\end{array}$ & $\begin{array}{c}\Delta_{\text {Shift }} \\
\left(\mathrm{cm}^{-1}\right)\end{array}$ \\
\hline 175 & 5026 & 5198 & 3 & 8.2 & 8.3 & 0.1 & 174.6 & 174.7 & 0.1 \\
\hline 190 & 1712 & 1680 & -2 & 4.7 & 4.7 & -0.1 & 190.3 & 190.3 & 0.0 \\
\hline 210 & 1246 & 1660 & 33 & 5.1 & 6.3 & 1.3 & 209.7 & 209.8 & 0.1 \\
\hline 265 & 8015 & 8172 & 2 & 8.4 & 8.3 & -0.1 & 265.4 & 265.4 & 0.0 \\
\hline 287 & 8325 & 7656 & -8 & 7.4 & 7.9 & 0.5 & 287.2 & 287.2 & 0.1 \\
\hline 313 & 11,860 & 10,815 & -9 & 10.1 & 9.8 & -0.3 & 313.0 & 313.2 & 0.2 \\
\hline 360 & 10,980 & 11,004 & 0 & 14.7 & 15.6 & 0.9 & 359.8 & 360.2 & 0.4 \\
\hline 400 & 4235 & 3636 & -14 & 19.8 & 18.7 & -1.1 & 400.0 & 400.1 & 0.1 \\
\hline 423 & 2571 & 2521 & -2 & 10.1 & 10.6 & 0.6 & 423.1 & 423.3 & 0.1 \\
\hline 514 & 3356 & 3115 & -7 & 9.1 & 8.5 & -0.6 & 514.4 & 514.4 & 0.0 \\
\hline 544 & 2150 & 1579 & -27 & 15.0 & 13.4 & -1.7 & 554.1 & 554.4 & 0.3 \\
\hline 691 & 8095 & 8214 & 1 & 17.7 & 18.1 & 0.4 & 691.5 & 691.6 & 0.1 \\
\hline 737 & 1117 & 2488 & 123 & 11.6 & 18.9 & 7.2 & 737.7 & 737.3 & -0.4 \\
\hline 783 & 19,738 & 20,040 & 2 & 15.3 & 16.7 & 1.3 & 783.2 & 784.0 & 0.9 \\
\hline 991 & 9999 & 8495 & -15 & 28.8 & 27.0 & -1.7 & 991.1 & 990.4 & -0.6 \\
\hline
\end{tabular}

One striking feature is that the previously intensity-sensitive modes assigned to the $\mathrm{TiO}_{6}$ octahedron are almost not responsive for the DW in context of the FWHM with one exception discussed below, while in z-face geometry, the opposite was often observed. Here, a change in FWHM, but not in intensity, is to be found. When a phonon features a directional dependent coupling (e.g., LO-phonons), it can be an indication that those phonons do offer polar parts in those directions.

The $991 \mathrm{~cm}^{-1}$ mode is the only vibration that is intensity- as well as FWHM-sensitive for DWs. The $v_{1}\left(\mathrm{PO}_{4}\right)$ vibration assigned peak stands out in contrast to the other changing modes for this parameter, since it is becoming narrower at the DW. Therefore, one may eliminate the influence of defects in this area, which would broaden the allowed selection rules and therefore also the peak.

However this broadening, which may be stoichiometric induced, is observable for the $360 \mathrm{~cm}^{-1}$ mode and $783 \mathrm{~cm}^{-1}$ mode. The first one is linked to the $v_{2}\left(\mathrm{PO}_{4}\right)$ vibration, whereas the second one is assigned to the $v_{3}\left(\mathrm{TiO}_{6}\right)$ vibration. It is not surprising that these phonons react differently, since the angular dispersion on the xy-surface is different than in the xz- or yz-surface. Therefore, there are also other shifts or changes in the FWHM.

The most sensitive modes are registered for the shift of the center frequency. While the $313 \mathrm{~cm}^{-1}$ mode is already striking for the intensity changes, the $360 \mathrm{~cm}^{-1}$ and $783 \mathrm{~cm}^{-1}$ peaks appear in the FWHM difference. A noticeable change in even both features is visible for the $991 \mathrm{~cm}^{-1}$ mode. Just the $738 \mathrm{~cm}^{-1}$ vibration is only sensitive for DWs via shifting. This mode is linked to the $v_{1}\left(\mathrm{TiO}_{6}\right)$ vibration. It is important to note, that there are two $\mathrm{PO}_{4}$ related vibrations, and two more certain $\mathrm{TiO}_{6}$ assigned vibrations. For both crystallographic structures a shift to higher as well as to lower wavenumbers can be observed. Such a shift in this case can often be related to local strain at the domain wall.

With respect to the assessed suitable fitting parameter for this scattering geometry, we present the resulting Raman images for the $783 \mathrm{~cm}^{-1} \mathrm{~B}_{1}$-TO mode on $\mathrm{y}$-cut KTP shown in Figure 5.

To analyze the domain evolution in the entire depth of the crystal (z-direction), a detailed visualization of the domain structure on the $y$-face was performed in $y(x, z) \bar{y}$ scattering geometry, whereby the the spatial variated peak frequency shift of the $\mathrm{B}_{1}-\mathrm{TO}$ phonon mode at $783 \mathrm{~cm}^{-1}$ was utilized. In this specific configuration, the peak frequency at domain walls is shifted to higher frequencies in the range of $0.8 \mathrm{~cm}^{-1}$ from the central frequency peak. Corresponding Raman images for three different regions are depicted in 
Figure 6: (a) a scan close to the $-\mathrm{z}$ surface $(\mathrm{z}=5-35 \mu \mathrm{m})$, (b) a scan around $300 \mu \mathrm{m}$ depth $(\mathrm{z}=300-330 \mu \mathrm{m})$, where the domain structure becomes inhomogeneous, and (c) a scan close to the $+z$ surface $(z=970$ to $1000 \mu \mathrm{m})$.

\section{Y-cut KTP: $783 \mathrm{~cm}^{-1}$ phonon-mode}
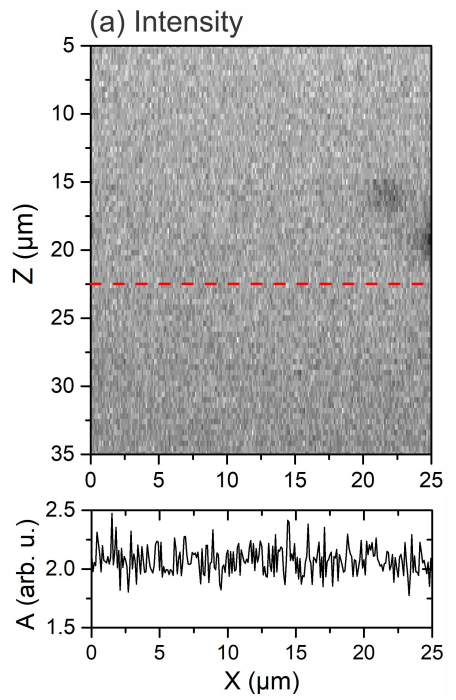
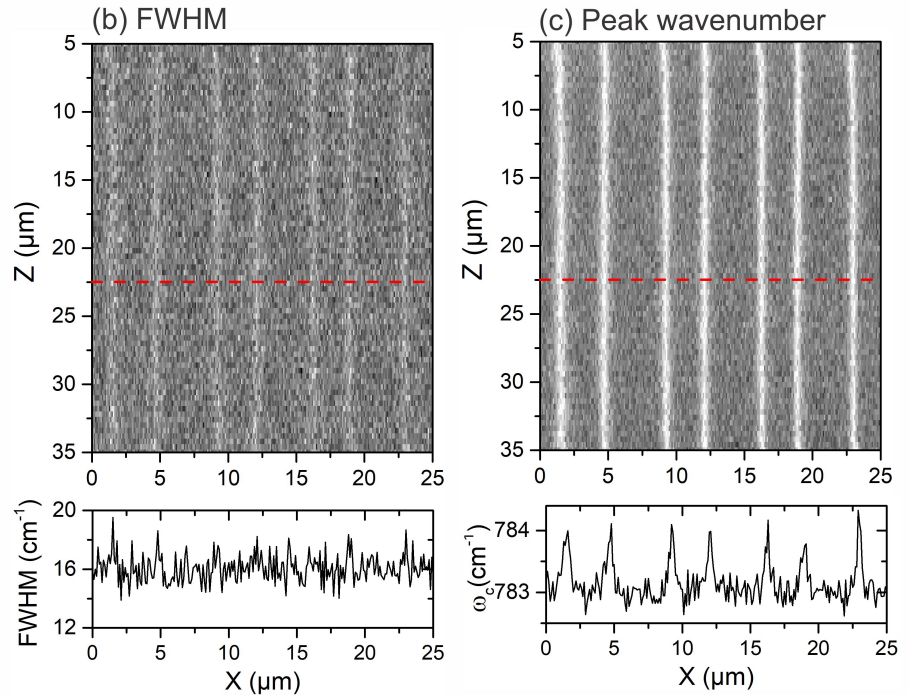

Figure 5. Raman images of the non-polar y-surface of a periodically poled KTP sample based on the variation of intensity (a), FWHM (b) and peak wave numbers (c) of the $783^{-1}$ phonon mode highlighted in Figure 4.

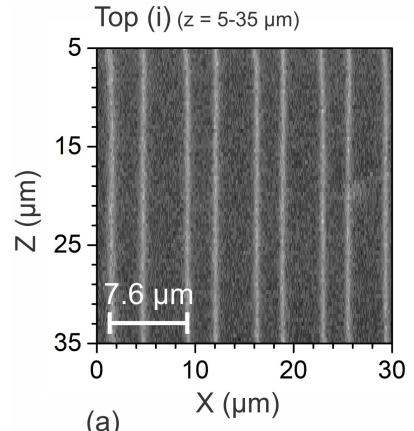

(a)

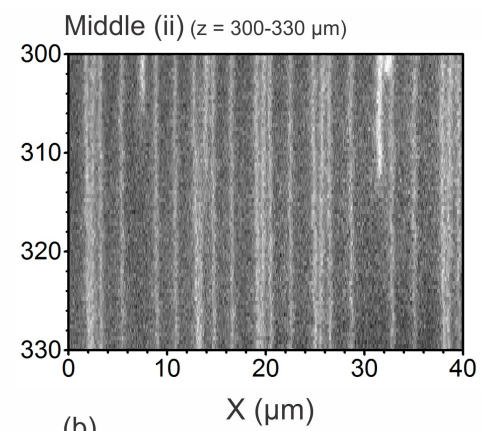

(b)

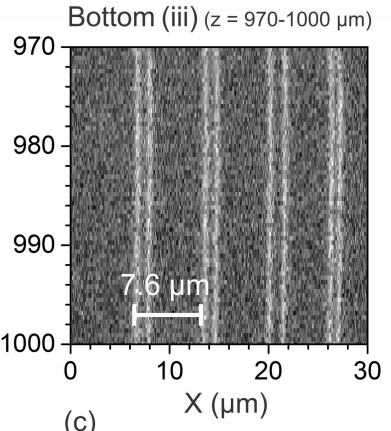

(c)
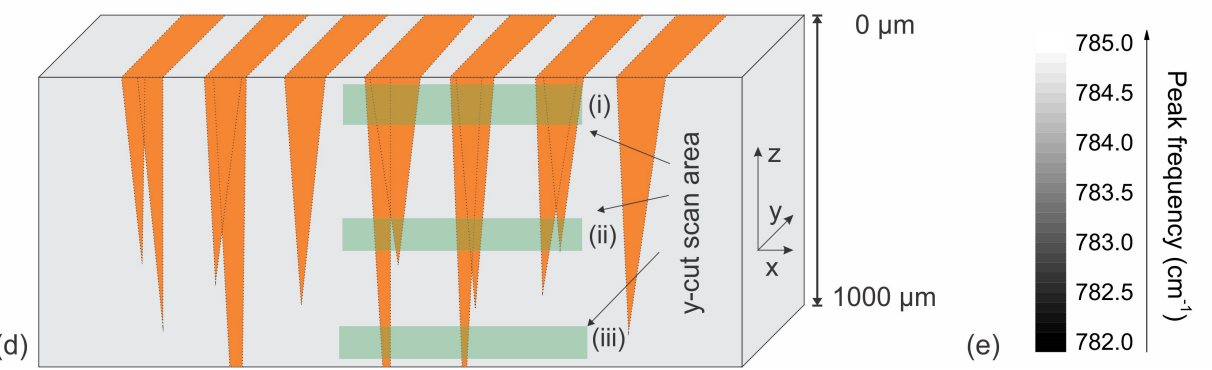

Figure 6. Detailed images of domain structures on $y$-cut $(y(x, z) \bar{y})$ measured in three areas of interest, (a) close to the -z surface, (b) in the region, where irregular domain structures appear and (c) close to the back side evaluated from the $783 \mathrm{~cm}^{-1}$ mode. A sketch of the measurement geometry is given in (d). A scale for the peak frequency with respect to the acquired Raman images $(\mathbf{a}-\mathbf{c})$ is to be found in (e). 
In principle over the entire depth, specific domain walls can be found. Additionally, over the complete scan range, not all inverted domains follow a path parallel to the $\mathrm{z}$ direction, but instead show some lateral movement on the order of a few $\mu \mathrm{m}$. Based on this data, three distinct regions can be subdivided: In the surface-near region (c.f. Figure 6a) the domain structure appears nearly to be homogeneous. This corresponds to the region, where the monitoring was performed resulting in a duty cycle of approximately 50/50 and a transferred domain period of $7.6 \mu \mathrm{m}$. A complex domain structure built up by various only partly merged spike-like domains can be found within the depth region from 300 to $330 \mu \mathrm{m}$ (c.f. Figure $6 \mathrm{~b}$ ). From the opening angle between $0.5^{\circ}$ to $1^{\circ}$ of this spike domain, it can be estimated that the domain velocity in the z-direction is at least 50 to 100 times faster compared to the domain velocity in the $\mathrm{x}$-direction. This is slightly lower than the previously measured ratio of about 200 to 250 by Canalias et al. [48]. A possible reason may be a different crystal stoichiometry in our crystals, as in particular the potassium stoichiometry is known to influence the material properties [49] and to vary between different crystals and even within the same wafer. This behavior can also be found in regions beyond this area. Close to the $z$-face (bottom of the sample, $z=970-1000 \mu \mathrm{m}$ ) the imaged domain structure (c.f. Figure $6 c$ ) shows a strongly altered duty cycle. However, the period of $7.6 \mu \mathrm{m}$ suggests that the inverted domains originally started their growth from a single nucleation side under adjacent electrodes. Note that the scan area for Figure $6 \mathrm{c}$ was especially chosen for inverted domains completely transferred to the bottom of the sample; there are also regions without a complete domain structure due to the merging of spike-like domains.

\section{Conclusions}

In this work, we have analyzed the vibrational properties in terms of the ferroelectric domain structure on the non-polar y-surface KTP. In this context, structure sensitive phonon modes have been assigned and based on this investigation we have demonstrated Raman imaging of the ferroelectric domain structure in y-face of KTP. Our analysis confirms, that our in situ poling monitoring method yields suitable domain structures close to the surface region and provides samples suitable for fabrication of periodically poled Rb-exchanged waveguides in KTP. For deeper regions we found spike-like merging of domains and change of the duty cycle to the bottom of the sample. All in all we demonstrated that the visualization of the domain structure on the non-polar y-face of KTP via Raman spectroscopy is also possible, and therefore enables a more elaborated improvement of the poling process and dynamics.

Author Contributions: Conceptualization, G.B. and M.R.; methodology, J.B. and M.R. and G.B.; software, J.B.; formal analysis, J.B. and M.R.; investigation, J.B. and P.W.M.M. and M.R.; writingoriginal draft preparation, J.B. and M.R. and P.W.M.M. and G.B.; writing - review and editing, J.B. and P.W.M.M. and M.R. and C.E. and L.P. and M.S. and C.S. and A.Z. and G.B.; visualization, P.W.M.M. and M.R.; project administration, C.S. and G.B.; funding acquisition, C.S. and A.Z. All authors have read and agreed to the published version of the manuscript.

Funding: This research was funded by the Deutsche Forschungsgemeinschaft (DFG) under Project No. 231447078 and by TRR 142 "Tailored Nonlinear Photonics" and by the Forschungsgruppe 5044.

Data Availability Statement: The data that support the findings of this study are contained within the article or are available from the corresponding author upon reasonable request.

Conflicts of Interest: The authors declare no conflict of interest. 


\begin{tabular}{|c|c|}
\hline \multicolumn{2}{|c|}{ Abbreviations } \\
\hline \multicolumn{2}{|c|}{ The following abbreviations are used in th } \\
\hline AFM & Atomic force microscopy \\
\hline CCD & Charged-coupled device \\
\hline DW & Domain wall \\
\hline FWHM & Full width at half maximum \\
\hline IR & Infrared \\
\hline KTP & Potassium titanyl phosphate \\
\hline $\mathrm{LN}$ & Lithium niobate \\
\hline LO & Longitudinal optical \\
\hline NA & Numerical aperture \\
\hline PDC & Parametric down conversion \\
\hline PFM & Piezoresponse force microscopy \\
\hline QPM & Quasi phase-matching \\
\hline TO & Transversal optical \\
\hline UV & Ultraviolet \\
\hline
\end{tabular}

\section{References}

1. Masse, R.; Grenier, J.C. Study of the monophosphates of type $\mathrm{M} 1 \mathrm{TiOPO}_{4}$ where M1=K, Rb and Ti. Bull. Soc. Fr. Mineral. Crystallogr. 1971, 94, 437. [CrossRef]

2. Zumsteg, F.C.; Bierlein, J.D.; Gier, T.E. KxRb1-xTiOPO 4 : A new nonlinear optical material. J. Appl. Phys. 1976, 47, 4980-4985. [CrossRef]

3. Bierlein, J.D.; Vanherzeele, H. Potassium titanyl phosphate: Properties and new applications. J. Opt. Soc. Am. B 1989, 6, 622. [CrossRef]

4. Canalias, C.; Pasiskevicius, V.; Clemens, R.; Laurell, F. Submicron periodically poled flux-grown KTiOPO . Appl. Phys. Lett. 2003, 82, 4233-4235. [CrossRef]

5. Canalias, C.; Pasiskevicius, V.; Laurell, F. Periodic Poling of KTiOPO 4: From Micrometer to Sub-Micrometer Domain Gratings. Ferroelectrics 2006, 340, 27-47. [CrossRef]

6. $\quad$ Eigner, C.; Santandrea, M.; Padberg, L.; Volk, M.F.; Rüter, C.E.; Herrmann, H.; Kip, D.; Silberhorn, C. Periodically poled ridge waveguides in KTP for second harmonic generation in the UV regime. Opt. Express 2018, 26, 28827. [CrossRef] [PubMed]

7. Canalias, C.; Pasiskevicius, V. Mirrorless optical parametric oscillator. Nat. Photonics 2007, 1, 459-462. [CrossRef]

8. Christ, A.; Eckstein, A.; Mosley, P.J.; Silberhorn, C. Pure single photon generation by type-I PDC with backward-wave amplification. Opt. Express 2009, 17, 3441-3446. [CrossRef]

9. Fejer, M.; Magel, G.; Jundt, D.; Byer, R. Quasi-phase-matched second harmonic generation: Tuning and tolerances. IEEE J. Quantum Electron. 1992, 28, 2631-2654. [CrossRef]

10. Karlsson, H.; Laurell, F.; Cheng, L.K. Periodic poling of $\mathrm{RbTiOPO}_{4}$ for quasi-phase matched blue light generation. Appl. Phys. Lett. 1999, 74, 1519. [CrossRef]

11. Wang, C.; Xiong, X.; Andrade, N.; Venkataraman, V.; Ren, X.F.; Guo, G.C.; Lončar, M. Second harmonic generation in nanostructured thin-film lithium niobate waveguides. Opt. Express 2017, 25, 6963. [CrossRef]

12. Li, G.; Chen, Y.; Jiang, H.; Chen, X. Broadband sum-frequency generation using d33 in periodically poled $\mathrm{LiNbO}_{3}$ thin film in the telecommunications band. Opt. Lett. 2017, 42, 939. [CrossRef]

13. Canalias, C.; Hirohashi, J.; Pasiskevicius, V.; Laurell, F. Polarization-switching characteristics of flux-grown $\mathrm{KTiOPO}_{4}$ and $\mathrm{RbTiOPO}_{4}$ at room temperature. J. Appl. Phys. 2005, 97, 124105. [CrossRef]

14. Karlsson, H.; Laurell, F. Electric field poling of flux grown $\mathrm{KTiOPO}_{4}$. Appl. Phys. Lett. 1997, 71, 3474-3476. [CrossRef]

15. Sorokina, N.I.; Voronkova, V.I. Structure and properties of crystals in the potassium titanyl phosphate family: A review. Crystallogr. Rep. 2007, 52, 80-93. [CrossRef]

16. Hellström, J.; Clemens, R.; Pasiskevicius, V.; Karlsson, H.; Laurell, F. Real-time and in situ monitoring of ferroelectric domains during periodic electric field poling of $\mathrm{KTiOPO}_{4}$. J. Appl. Phys. 2001, 90, 1489-1495. [CrossRef]

17. Canalias, C.; Pasiskevicius, V.; Fragemann, A.; Laurell, F. High-resolution domain imaging on the nonpolar y-face of periodically poled $\mathrm{KTiOPO}_{4}$ by means of atomic force microscopy. Appl. Phys. Lett. 2003, 83, 734. [CrossRef]

18. Wittborn, J.; Canalias, C.; Rao, K.V.; Clemens, R.; Karlsson, H.; Laurell, F. Nanoscale imaging of domains and domain walls in periodically poled ferroelectrics using atomic force microscopy. Appl. Phys. Lett. 2002, 80, 1622-1624. [CrossRef]

19. Johann, F.; Ying, Y.J.; Jungk, T.; Hoffmann, Á.; Sones, C.L.; Eason, R.W.; Mailis, S.; Soergel, E. Depth resolution of piezoresponse force microscopy. Appl. Phys. Lett. 2009, 94, 172904. [CrossRef]

20. Ya Shur, V.; Zelenovskiy, P.S.; Shur, V.Y.; Zelenovskiy, P.S. Micro- and nanodomain imaging in uniaxial ferroelectrics: Joint application of optical, confocal Raman, and piezoelectric force microscopy. J. Appl. Phys. 2014, 116, 066802. [CrossRef]

21. Rüsing, M.; Eigner, C.; Mackwitz, P.; Berth, G.; Silberhorn, C.; Zrenner, A. Identification of ferroelectric domain structure sensitive phonon modes in potassium titanyl phosphate: A fundamental study. J. Appl. Phys. 2016, 119, 044103. [CrossRef] 
22. Flörsheimer, M.; Paschotta, R.; Kubitscheck, U.; Brillert, C.; Hofmann, D.; Heuer, L.; Schreiber, G.; Verbeek, C.; Sohler, W.; Fuchs, H. Second-harmonic imaging of ferroelectric domains in LiNbO 3 with micron resolution in lateral and axial directions. Appl. Phys. B Lasers Opt. 1998, 67, 593-599. [CrossRef]

23. Bozhevolnyi, S.I.; Hvam, J.M.; Pedersen, K.; Laurell, F.; Karlsson, H.; Skettrup, T.; Belmonte, M. Second-harmonic imaging of ferroelectric domain walls. Appl. Phys. Lett. 1998, 73, 1814-1816. [CrossRef]

24. Spychala, K.J.; Mackwitz, P.; Widhalm, A.; Berth, G.; Zrenner, A. Spatially resolved light field analysis of the second-harmonic signal of $\chi(2)$-materials in the tight focusing regime. J. Appl. Phys. 2020, 127, 023103. [CrossRef]

25. Spychala, K.J.; Mackwitz, P.; Ruesing, M.; Widhalm, A.; Berth, G.; Silberhorn, C.; Zrenner, A. Nonlinear focal mapping of ferroelectric domain walls in $\mathrm{LiNbO}_{3}$ : Analysis of the SHG microscopy contrast mechanism. J. Appl. Phys. 2020, 128, 234102. [CrossRef]

26. Berth, G.; Quiring, V.; Sohler, W.; Zrenner, A. Depth-Resolved Analysis of Ferroelectric Domain Structures in Ti:PPLN Waveguides by Nonlinear Confocal Laser Scanning Microscopy. Ferroelectrics 2007, 352, 78-85. [CrossRef]

27. Kämpfe, T.; Reichenbach, P.; Schröder, M.; Haußmann, A.; Eng, L.M.; Woike, T.; Soergel, E. Optical three-dimensional profiling of charged domain walls in ferroelectrics by Cherenkov second-harmonic generation. Phys. Rev. B 2014, 89, 035314. [CrossRef]

28. Ruesing, M.; Zhao, J.; Mookherjea, S. Second harmonic microscopy of poled $\mathrm{x}$-cut thin film lithium niobate: Understanding the contrast mechanism. J. Appl. Phys. 2019, 126, 1-14. [CrossRef]

29. Rüsing, M.; Sanna, S.; Neufeld, S.; Berth, G.; Schmidt, W.G.; Zrenner, A.; Yu, H.; Wang, Y.; Zhang, H. Vibrational properties of LiNb1-xTaxO3 mixed crystals. Phys. Rev. B 2016, 93, 184305. [CrossRef]

30. Fontana, M.D.; Bourson, P. Microstructure and defects probed by Raman spectroscopy in lithium niobate crystals and devices. Appl. Phys. Rev. 2015, 2, 040602. [CrossRef]

31. Cherifi-Hertel, S.; Bulou, H.; Hertel, R.; Taupier, G.; Dorkenoo, K.D.H.; Andreas, C.; Guyonnet, J.; Gaponenko, I.; Gallo, K.; Paruch, P. Non-Ising and chiral ferroelectric domain walls revealed by nonlinear optical microscopy. Nat. Commun. 2017, 8, 15768. [CrossRef] [PubMed]

32. Cherifi-Hertel, S.; Voulot, C.; Acevedo-Salas, U.; Zhang, Y.; Cregut, O.; Dorkenoo, K.; Hertel, R. Shedding light on non-Ising polar domain walls: Insight from second harmonic generation microscopy and polarimetry analysis. J. Appl. Phys. 2021, $129,081101$. [CrossRef]

33. Padberg, L.; Santandrea, M.; Rüsing, M.; Brockmeier, J.; Mackwitz, P.; Berth, G.; Zrenner, A.; Eigner, C.; Silberhorn, C. Characterisation of width-dependent diffusion dynamics in rubidium-exchanged KTP waveguides. Opt. Express 2020, 28, 24353. [CrossRef] [PubMed]

34. Rüsing, M.; Neufeld, S.; Brockmeier, J.; Eigner, C.; Mackwitz, P.; Spychala, K.; Silberhorn, C.; Schmidt, W.G.; Berth, G.; Zrenner, A.; et al. Imaging of 180 ferroelectric domain walls in uniaxial ferroelectrics by confocal Raman spectroscopy: Unraveling the contrast mechanism. Phys. Rev. Mater. 2018, 2, 103801. [CrossRef]

35. Eigner, C.; Padberg, L.; Santandrea, M.; Herrmann, H.; Brecht, B.; Silberhorn, C. Spatially single mode photon pair source at $800 \mathrm{~nm}$ in periodically poled Rubidium exchanged KTP waveguides. Opt. Express 2020, 28, 32925. [CrossRef]

36. Fatuzzo, E.; Merz, W.J. Ferroelectricity; North-Holland: Amsterdam, The Netherlands, 1967.

37. Shur, V.Y.; Akhmatkhanov, A.R.; Baturin, I.S. Micro- and nano-domain engineering in lithium niobate. Appl. Phys. Rev. 2015, 2, 040604. [CrossRef]

38. Ansari, V.; Roccia, E.; Santandrea, M.; Doostdar, M.; Eigner, C.; Padberg, L.; Gianani, I.; Sbroscia, M.; Donohue, J.M.; Mancino, L.; et al. Heralded generation of high-purity ultrashort single photons in programmable temporal shapes. Opt. Express 2018, 26, 2764. [CrossRef]

39. Berth, G.; Hahn, W.; Wiedemeier, V.; Zrenner, A.; Sanna, S.; Schmidt, W.G. Imaging of the Ferroelectric Domain Structures by Confocal Raman Spectroscopy. Ferroelectrics 2011, 420, 44-48. [CrossRef]

40. Gopalan, V.; Dierolf, V.; Scrymgeour, D.A. Defect-Domain Wall Interactions in Trigonal Ferroelectrics. Annu. Rev. Mater. Res. 2007, 37, 449-489. [CrossRef]

41. Jach, T.; Kim, S.; Gopalan, V.; Durbin, S.; Bright, D. Long-range strains and the effects of applied field at $180^{\circ}$ ferroelectric domain walls in lithium niobate. Phys. Rev. B 2004, 69, 064113. [CrossRef]

42. Dierolf, V.; Sandmann, C. Inspection of periodically poled waveguide devices by confocal luminescence microscopy. Appl. Phys. B 2004, 78, 363-366. [CrossRef]

43. Zhang, Y.; Guilbert, L.; Bourson, P. Characterization of Ti: $\mathrm{LiNbO}_{3}$ waveguides by micro-raman and luminescence spectroscopy. Appl. Phys. B 2004, 78, 355-361. [CrossRef]

44. Stone, G.; Knorr, B.; Gopalan, V.; Dierolf, V. Frequency shift of Raman modes due to an applied electric field and domain inversion in LiNbO3. Phys. Rev. B 2011, 84, 134303. [CrossRef]

45. Fontana, M.D.; Hammoum, R.; Bourson, P.; Margueron, S.; Shur, V.Y. Raman Probe on PPLN Microstructures. Ferroelectrics 2008, 373, 26-31. [CrossRef]

46. Stone, G.; Dierolf, V. Influence of ferroelectric domain walls on the Raman scattering process in lithium tantalate and niobate. Opt. Lett. 2012, 37, 1032. [CrossRef] [PubMed]

47. Rüsing, M. In Depth Raman Analysis of the Ferroelectrics $\mathrm{KTiOPO}_{4}$ and $\mathrm{LiNbO}_{3}$ - Role of Domain Boundaries and Defects. Ph.D. Thesis, University of Paderborn, Paderborn, Germany, 2018. [CrossRef] 
48. Canalias, C. Domain Engineering in $\mathrm{KTiOPO}_{4}$. Ph.D. Thesis, Royal Institute of Technology Stockholm, Stockholm, Sweden, 2005.

49. Roth, M. Stoichiometry and Domain Structure of KTP-Type Nonlinear Optical Crystals. In Springer Handbook of Crystal Growth; Springer: Berlin/Heidelberg, Germany, 2010; pp. 691-723. [CrossRef] 